\title{
Study of Ellipsometry of Swelling of Styrene-acrylic Bentonite-containing Copolymer Composites
}

\author{
Ganiyev Bakhtiyor Shukurullayevich \\ Department of Chemistry, Faculty of Natural Science, Bukhara State University, Bukhara City, Uzbekistan
}

\section{Email address:}

b.ganiyev1990@gmail.com

\section{To cite this article:}

Ganiyev Bakhtiyor Shukurullayevich. Study of Ellipsometry of Swelling of Styrene-acrylic Bentonite-containing Copolymer Composites. Science Journal of Chemistry. Special Issue: Hybrid Organic-Inorganic Materials, from Fundamentals to Practical Applications.

Vol. 8, No. 3, 2020, pp. 72-76. doi: 10.11648/j.sjc.20200803.15

Received: March 25, 2020; Accepted: June 10, 2020; Published: June 28, 2020

\begin{abstract}
In this work, the study of copolymer acrylic films was carried out on a laser photoelectric ellipsometer LEF-ZM-1. In this work, we consider a chemical sensor based on an integrated optical sensing element, propose structural schemes of IOCS, as well as conduct studies on a simulation model and assess the potential for detecting changes in the concentration of various substances. For this, in the course of the work, we studied the change in the refractive indices of polymer films with a Navbahor bentonite content of $5 \mathrm{wt} . \%$, Depending on the time of their swelling in aqueous solutions containing $\mathrm{Cr}$ ions. The most important characteristics for acrylic copolymers are mechanical strength and sorption, since it is the values of these parameters that impose significant restrictions on the field of their application. Therefore, in this article, in addition to ellipsometry, the effect of the initiator concentration on the absorption properties of polymer composites and the influence of the synthesis temperature on the absorption properties of copolymer composites containing Navbahor bentonite are also studied. During the study, it was clarified that acrylic mineral-containing composites obtained at $42^{\circ} \mathrm{C}$ have the maximum sorption ability.
\end{abstract}

Keywords: Ellipsometry, Bentonite, Styrene, Sensor, Refraction, Compensator, Composite

\section{Introduction}

Current trends in the development of thin-film technologies and the semiconductor industry inevitably lead to a decrease in the characteristic dimensions of the created structures [1-4]. This places high demands on analytical tools for controlling the parameters of layered structures during their production: composition of layers, crystalline perfection of materials, and primarily their geometric characteristics - layer thicknesses. There is an extensive arsenal of methods for such control: Auger spectroscopy, diffraction of slow and fast electrons, Xray photoelectron spectroscopy, atomic force microscopy, and others. A special place in this series is ellipsometry [5-7]. This is an optical method based on an analysis of the polarization state of light reflected from a sample. It is used to study the physicochemical properties of a surface, its morphology, to measure the thickness of multilayer structures and characterize the optical properties of thin films. A number of significant advantages of this method make it extremely attractive.

Ellipsometry has a number of invaluable advantages over many research methods, concerning not only its sensitivity, but also the range of materials covered by this method, and the ease of preparing samples for research. The experimenter is presented with the possibility of continuous observation of physical or chemical processes on the surface, the possibility of repeated experience on the same sample [8-12].

The unique features of ellipsometry, according to the literature, are not fully used. Ellipsometry applications are not exhausted, many new experiments can be put in the form of our work.

\section{Materials and Research Methods}

For the synthesis of acrylic copolymers the following are used starting material:

1. Styrene;

2. Acrylamide;

3. Hydroxide;

4. N, N' - methylene bis acrylamide;

5. Tetramethylethylenediamine;

6. Ammonium persulfate (PSA)

7. Dinitrile azobisisobutyric acid (DAC).

Styrene should be purified from the inhibitor. For this 
distillation is used at $+38-+43^{\circ} \mathrm{C}$ and a pressure of 8-12 $\mathrm{mmHg}$. art. water jet pump.

$\mathrm{N}, \mathrm{N}^{\prime}$ - methylene bis acrylamide is purified using recrystallization of ethyl alcohol. Hot saturated at $40^{\circ} \mathrm{Ca}$ solution of $1 \mathrm{~g}$ of $\mathrm{N}, \mathrm{N}^{\prime}$ - methylene bis acrylamide is dissolved in $5 \mathrm{ml}$ of ethyl alcohol and filtered on a funnel for hot filtering. Precipitate white flake crystals are dried by vacuum drying or in thermostat. The dried crystals are stored either in a bux or in a desiccator. Since the purity of PSA has an impact on the physico-mechanical and sorption properties will also require recrystallization of the PSA. Poly (styreneco-acrylamide) and polystyrene polymers were synthesized by boiling temperature soap free emulsion polymerization in aqueous medium with ammonium persulfate as the initiator. To a mixture of styrene and acrylamide in 1: 1 ratio, the cross linker (N, N-methylene-bis-acrylamide) and initiator (ammonium persulfate) were added and the mixture was heated at $70^{\circ} \mathrm{C}$ in an electric oven for $1 \mathrm{hr}$. The co-polymeric material so formed was washed with distilled water and cut into small uniform pieces. The resulting polymers were dissolved in dimethylformamide and teterahydrofuran (DMF: THF) (4: 1) to form polymer [13-17].

Bentonites were used as fillers. Bentonite was provided by the Navbahor Deposit of the Republic of Uzbekistan. One of the most important deposits of bentonite clays in Uzbekistan is considered to be the Navbahor Deposit in Navoi region. Unfortunately, the thickening, colloidal-chemical and sorption properties of clays of this Deposit have not been studied yet.

The most attractive sorbents from waste vegetable raw materials. Practically unlimited reserves of these materials, their cheapness, simple production technology, environmental safety of the processes of processing used sorbents, as well as fairly high adsorption, ion exchange and filtration properties of sorbents stimulate research aimed at obtaining new adsorption-active materials from plant raw materials. For this purpose, mechanical and chemical modification of natural bentonite with styrene acrylamide copolymers was carried out. The dependences of the amount of grafted copolymer on the monomer content in the system and the duration of the process are investigated. Modification of minerals was performed depending on the duration of the process, the ratio of components. The amount of polymer grafting ranged from 3.5 to $6.2 \%$.

We have studied the possibility of replacing imported water-absorbing sorbents of domestic production based on alkaline bentonite of the Navbahor Deposit and styreneacrylamide copolymers [18-20].

The ellipsometry method is based on the fact that by analyzing the characteristics of elliptical polarization, one can obtain information on the optical constants of reflecting (refractive) materials, surface layers and films [21].

The first step is to determine the parameters of the polarization ellipse $\Delta$ and $\psi$. Elliptical polarization analysis is performed using ellipsometers. Schematically (Figure 1), the ellipsometer is a two-arm optical device in which the light of the source $\mathrm{S}$, passing through the monochromatic filter $\mathrm{F}$ and the collimator $\mathrm{C}$, is linearly polarized by the prism $\mathrm{P}$, reflected from the sample $M$ passes through the analyzer $A$ (which is a linearly polarizing device similar to P) and is recorded either visually or photoelectrically (D). A compensator $\mathrm{K}$ is installed between the polarizer $\mathrm{P}$ and analyzer A, which changes the phase shift between the $\mathrm{p}$ and s components accordingly [22].

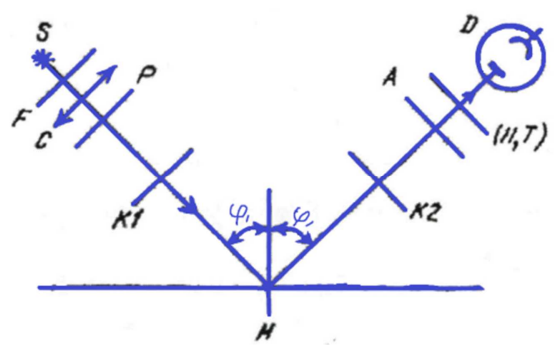

Figure 1. Layout of the elements of the ellipsometer when working in reflected light.

The orientation of the elements $\mathrm{P}, \mathrm{K}, \mathrm{A}$ is set using the azimuth, which, by definition, is equal to the angle between the plane of incidence and the characteristic direction of this element, counted counterclockwise by the observer looking towards the beam; for $\mathrm{R}$ and $\mathrm{A}$, the directions of transmission serve as such directions; for the compensator of Senarmon, the F-direction. All polarizing elements are mounted in graduated frames for measuring azimuths [4, 23, 24].

The compensator can be installed both before and after the sample. In schemes with manual control or with servo drives, measurements of the parameters of the polarization ellipse are carried out by rotating two of the three polarizing elements with a fixed third element.

In this work, the study of copolymer acrylic films was carried out on a laser photoelectric ellipsometer LEF-ZM-1.

As mentioned above, one of the promising types of chemical sensors is integrated optical chemical sensors.

\section{Results and Discussions}

\subsection{Effect of Synthesis Temperature on Absorption Properties Copolymer Composites Containing Navbahor Bentonite}

Particular attention is paid to crosslinked acrylic polyelectrolytes, which are also called super moisture absorbents or hydrogels. The main characteristics of acrylic hydrogels necessary for practical use are a high degree of swelling and acceptable physical and mechanical properties.

As can be seen from the figure, the dependence of the equilibrium degree of swelling based on bentonite (Navbahor deposit) containing styrene-acrylic copolymer of composites on the experimental temperature is extreme. Samples obtained at a temperature of $30^{\circ} \mathrm{C}$ have a minimum degree of swelling. A decrease in sorption characteristics with a decrease in the synthesis temperature is explained by the fact that the limiting degree of monomer conversion decreases.

Acrylic mineral-containing composites obtained at $42^{\circ} \mathrm{C}$ possess the maximum sorption ability. With increasing polymerization temperature higher than $60^{\circ} \mathrm{C}$, the degree of 
swelling of acrylic copolymers decreases, which can be explained by the formation of more defective structure of the polymer network [25].

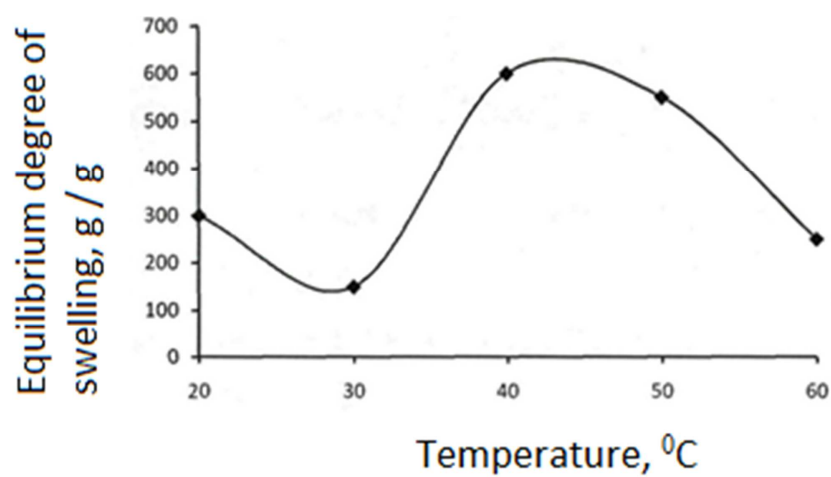

Figure 2. The dependence of the equilibrium degree of swelling of the polymer composition with a mass fraction of bentonite $30 \mathrm{wt} \%$ from the synthesis temperature.

\subsection{Effect of Initiator Concentration on Absorption Properties Polymer Composites}

The most important characteristics for acrylic copolymers are mechanical strength and sorption, since it is the values of these parameters that impose significant restrictions on the field of their application.

The figure shows a graph characterizing the sorption capabilities of bentonite (Navbahor deposit) -containing styreneacrylic copolymer compositions when they swell in distilled water at a temperature of $30^{\circ} \mathrm{C}$ depending on the concentration of the initiator - DAC (dinitrile azobisisobutyric acid).

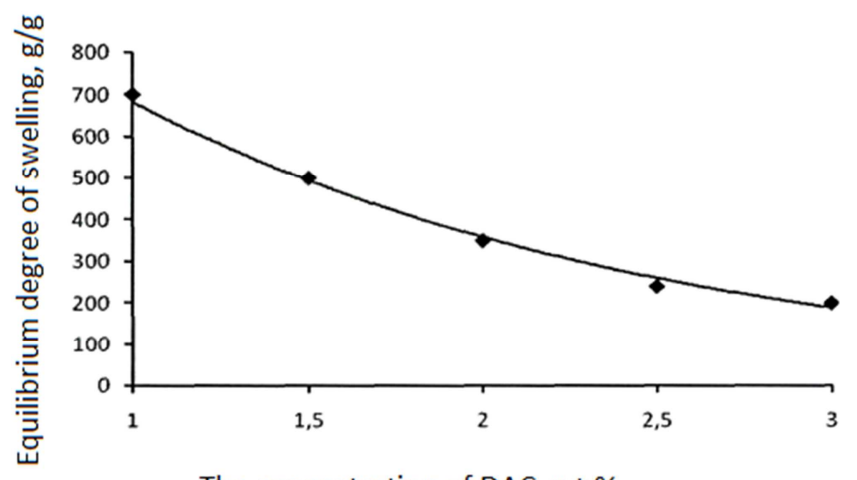

The concentration of DAC, wt.\%

Figure 3. The dependence of the equilibrium degree of swelling of acrylic a polymer composition with a mass fraction of bentonite of $30 \mathrm{wt} . \%$ from the concentration of initiator.

As can be seen from Figure 3, an increase in the initiator concentration of more than $0.5 \mathrm{wt} . \%$ Leads to a decrease in the equilibrium degree of swelling by more than 2 times. This can be explained by the fact that the formation of a copolymer with a reduced molecular weight between the crosslinking sites [26].

\subsection{Study of the Surface of Copolymer-Bentonite Composites with an Electron Microscope}

When studying the behavior of ionogenic crosslinked copolymers in aqueous electrolyte solutions, most often, two modes of interaction of a hydrogel with polyvalent metal ions are considered in the case when the latter form waterinsoluble compounds with reactive network groups:

- if the polymer concentration in the hydrogel-solution system is greater than the salt concentration, then the hydrogel absorbs predominantly pure water, increasing the salt concentration in the environment;

- if the polymer concentration is significantly lower than the salt concentration, then the hydrogel acts as an ionexchange sorbent.

In order to study both modes in this work, we studied the effect of an aqueous solution of chromium chloride, taken in a concentration range of 10-5-10-2 mol/L, on the absorption properties of synthesized composite bentonite-containing materials.

The study of the surface of polymer - bentonite composites with different proportions of bentonite in the composition of the polymer composition was performed on a Phillips XL 30 transmission electron microscope and the obtained micrographs are presented in Figure 4.

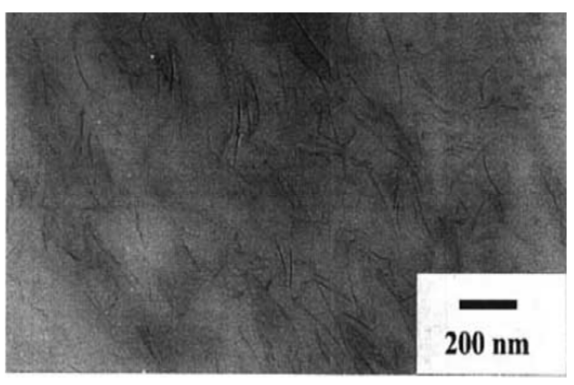

a)

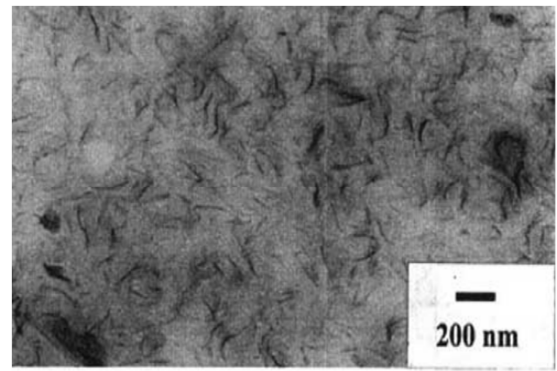

b)

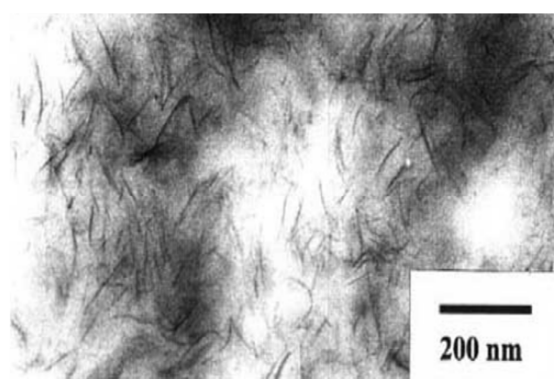

c)

Figure 4. Photos of the surface of composites based on acrylic copolymer and bentonite: a) unfilled copolymer; b) a composite with a concentration of bentonite of $10 \mathrm{wt} . \%$; c) a composite with a concentration of bentonite of 40 $w t . \%$. 
As can be seen from Figure 4, when nanoparticles are introduced during the synthesis, a partially ordered structure such as a house of cards is obtained, which was also observed in [27] when studying the polymerization of acrylamide with bentonite in an aqueous medium at a synthesis temperature of $40^{\circ} \mathrm{C}$ during the day.

\subsection{Ellipsometry Study of Swelling of Styrene-acrylic Bentonite-containing Films}

In this paper, we consider a chemical sensor based on an integrated optical sensing element, propose IOCS structural schemes, conduct studies on a simulation model and assess the potential for detecting changes in the concentration of various substances.

For this, in the course of the work, we studied the change in the refractive indices of polymer films with a Navbahor bentonite content of $5 \mathrm{wt} . \%$, Depending on the time of their swelling in aqueous solutions containing $\mathrm{Cr}$ ions. The results are presented in figures 5-6 [13, 23].

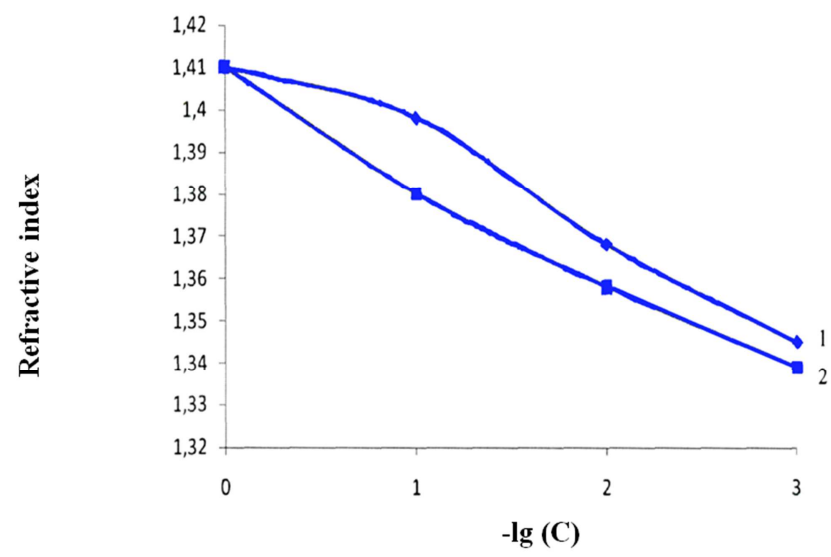

Figure 5. Graph of the dependence of the refractive index of the polymer film on the concentration of the solution (-lg C) $\mathrm{CrCl}_{3}, 1-2 \mathrm{~min}, 2-10 \mathrm{~min}$.

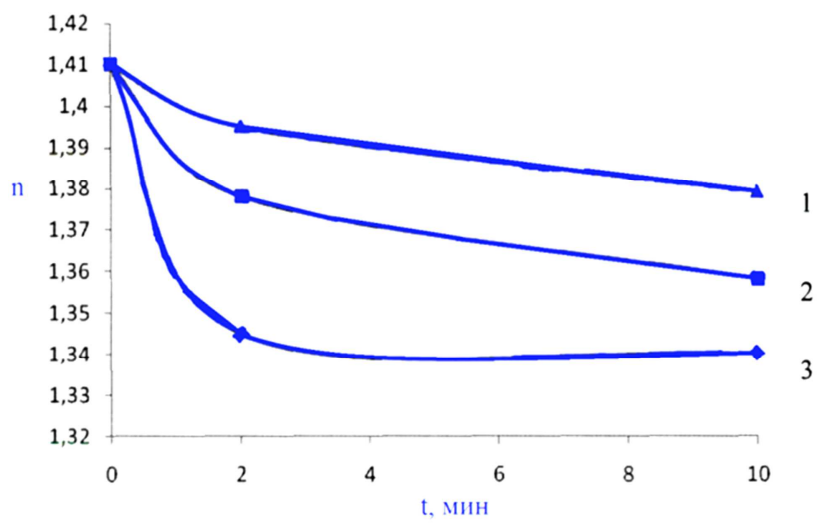

Figure 6. Graph of the refractive index of a polymer film of a bentonitecontaining composition with a bentonite fraction of $5 \mathrm{wt} \%$ From the time spent in an aqueous solution of $\mathrm{CrCl}_{3}, 1-0.1 \mathrm{M}, 2-0.01 \mathrm{M}, 3-0.001 \mathrm{M}$.

Tables 1-3 show the dependences of the refractive indices of the polymer film of the Navbahor bentonite-containing composition on the time spent in an aqueous solution of $\mathrm{CrCl}_{3}$.
Table 1. The refractive index of a solution of ions of $0,1 \mathrm{M} \mathrm{CrCl}$

\begin{tabular}{llll}
\hline \multicolumn{2}{l}{$\begin{array}{l}\text { A sample placed in a solution for } \\
\mathbf{2} \text { minutes }\end{array}$} & $\begin{array}{l}\text { A sample placed in a solution for } \\
\mathbf{1 0} \text { minutes }\end{array}$ \\
\hline 1 & $\mathrm{n}=1,399$ & 1 & $\mathrm{n}=1.378$ \\
2 & $\mathrm{n}=1,393$ & 2 & $\mathrm{n}=1,385$ \\
3 & $\mathrm{n}=1,399$ & 3 & $\mathrm{n}=1,373$ \\
4 & $\mathrm{n}=1,397$ & 4 & $\mathrm{n}=1,379$ \\
$\mathrm{n}_{0,1 * 2} \mathrm{CrCl}_{3}=1,397$ & & $\mathrm{n}_{0,1 * 10} \mathrm{CrCl}_{3}=1,379$ \\
\hline
\end{tabular}

Refractive index of the solution $\mathrm{n}_{0,1} \mathrm{CrCl}_{3}=1,3365$

Table 2. The refractive index of a solution of ions of $0,01 \mathrm{M} \mathrm{CrCl}_{3}$.

\begin{tabular}{llll}
\hline \multicolumn{2}{l}{$\begin{array}{l}\text { A sample placed in a solution for } \\
\mathbf{2} \text { minutes }\end{array}$} & $\begin{array}{l}\text { A sample placed in a solution for } \\
\mathbf{1 0} \text { minutes }\end{array}$ \\
\hline 1 & $\mathrm{n}=1,361$ & 1 & $\mathrm{n}=1.360$ \\
2 & $\mathrm{n}=1,366$ & 2 & $\mathrm{n}=1,355$ \\
3 & $\mathrm{n}=1,376$ & 3 & $\mathrm{n}=1,357$ \\
4 & $\mathrm{n}=1,3858$ & 4 & $\mathrm{n}=1,358$ \\
$\mathrm{n}_{0,01 * 2} \mathrm{CrCl}_{3}=1,368$ & $\mathrm{n}_{0,01 * 10} \mathrm{CrCl}_{3}=1,3575$ \\
\hline
\end{tabular}

Refractive index of the solution $\mathrm{n}^{0,1} \mathrm{CrCl}_{3}=1,3382$

Table 3. The refractive index of a solution of ions of $0,001 \mathrm{M} \mathrm{CrCl}_{3}$.

\begin{tabular}{|c|c|c|c|}
\hline \multicolumn{2}{|c|}{$\begin{array}{l}\text { A sample placed in a solution for } \\
2 \text { minutes }\end{array}$} & \multicolumn{2}{|c|}{$\begin{array}{l}\text { A sample placed in a solution for } \\
10 \text { minutes }\end{array}$} \\
\hline 1 & $\mathrm{n}=1,340$ & 1 & $\mathrm{n}=1.339$ \\
\hline 2 & $\mathrm{n}=1,340$ & 2 & $\mathrm{n}=1,339$ \\
\hline 3 & $\mathrm{n}=1,350$ & 3 & $\mathrm{n}=1,339$ \\
\hline 4 & $\mathrm{n}=1,343$ & 4 & $\mathrm{n}=1,339$ \\
\hline \multicolumn{2}{|c|}{$\mathrm{n}_{0,001 * 2} \mathrm{CrCl}_{3}=1,343$} & \multicolumn{2}{|c|}{$\mathrm{n}_{0,001 * 10} \mathrm{CrCl}_{3}=1,339$} \\
\hline
\end{tabular}

Refractive index of the solution $\mathrm{n}_{0,1} \mathrm{CrCl}_{3}=1,339$

As can be seen from figure 2, when the concentration of the $\mathrm{CrCl}_{3}$ solution increases from $0,001 \mathrm{M}$ to $0.1 \mathrm{M}$, the refractive index increases from 1,3365 to 1,37 and approaches the value of 1,339 , which corresponds to the refractive index of the salt solution with the initial concentration.

This can be explained by sorption of heavy metal ions on films. At the same time, we can talk about the prospects of using bentonite-containing material as the basis for creating a chemical sensor.

\section{Conclusion}

It can be concluded that the obtained bentonite-containing copolymer composites can be used in medicine and as a basis for integrated optical chemical sensors.

The study shows with an increase in the initiator concentration of more than $0.5 \mathrm{wt} . \%$ Leads to a decrease in the equilibrium degree of swelling by more than 2 times.

During the study, it was clarified that acrylic mineralcontaining composites obtained at $42^{\circ} \mathrm{C}$ have the maximum sorption ability. With an increase in the polymerization temperature higher than $60{ }^{\circ} \mathrm{C}$, the degree of swelling of the acrylic copolymers decreases, which can be explained by the formation of a more defective structure of the polymer network.

\section{Acknowledgements}

The authors are grateful to Andreas Furchner Professor of 
the Technischen Universität Berlin of Germany, Professor of the National University of Uzbekistan Mukhammadiyev Mukhtorjon Ganiyevich and the staff of the Institute of General and Inorganic Chemistry of the Academy of Sciences of Uzbekistan for their practical assistance in the preparation of the article.

\section{References}

[1] T. Oshige, T. Yamada, and A. Kazama, "Measuring Method for Ellipsometric Parameters and Ellipsometer," US Patent No. 5, 311, 285 (May 10, 1994).

[2] Collins R. W. et al. Advances in multichannel spectroscopic ellipsometry //Thin Solid Films. - 1998. - T. 313. - C. 18-32.

[3] Hu Z. G., Huang Z. M., Wu Y. N., Wang G. S., Meng X. J., Shi F. W., Chu J. H. //J. Vac. Sci. Technol. A, 2004. V. 22. № 4. P. 1152.

[4] Miao L., Jiang T., Tanemura S., et al. // Phys. Stat. Sol. (c), 2008. V. 5. № 5. P. 1125.

[5] Tompkins H., Irene E. A. Handbook of ellipsometry. William Andrew, 2005.

[6] Furchner, A.; Bittrich, E.; Rauch, S.; Uhlmann, P.; Hinrichs, K., "Temperature-Sensitive Swelling Behavior of Thin Poly (N-isopropylacrylamide) Brushes Studied by In-situ Infrared Spectroscopic Ellipsometry," Polymeric Materials: Science \& Engineering 2012, 107, 372- 373.

[7] Hinrichs, K.; Bittrich, E.; Aulich, D.; Furchner, A.; Minko, S.; Luzinov, I.; Stamm, M.; Uhlmann, P.; Eichhorn, K.-J., "Ellipsometry for Study of Smart Polymer Brushes," Polymeric Materials: Science \& Engineering 2012, 107, 655656.

[8] Ellipsometry-method of surface investigation / Ed. A. V. Rzhanov, "Science", Novosibirsk, 1983.

[9] Ellipsometry: theory, methods, applications. / Editors K. K. Svitashev, A. S. Mardezhov, Nauka, Novosibirsk, 1991.

[10] Gorshkov, M. M. Ellipsometry / M. M. Gorshkov. - M.: Sovetskoe radio, 1974. - P. 200

[11] Aseev A. L. / / Russian nanotechnologies, 2006. Vol. 1. No. 1, 2. P. 97.

[12] Rzhanov A. V, Svitashev K. K, Semenenko A. I, Semenenko L. V, Sokolov V. K. Basics of ellipsometry. Novosibirsk, Nauka, 1979, P. 424

[13] Ganiyev B. Sh., Sharipov M. S. To study the effect of temperature and time on the yield of styrene forming a sopolimer with acrylamide. Materials of the Republican scientific-practical conference "Actual problems of Chemical Science and innovative technologies in its teaching". Tashkent 2016 y. - P. 186-187.

[14] Ganyiev B. Sh., Sharipov M. S. Synthesis and Characterization of Poly (styrene-co-acrylamide) Polymers. Republican scientific and Practical Conference of young scientists Part II. Termiz, 2017 y. - P. 128.

[15] Patil, N. S. et al, 1996. Macroporous poly (sucrose acrylate) hydrogels for controlled release of macromolecules. Biomaterials 17, 2343-2350.
[16] Lee, W. Effect of bentonite on physical Properties and DrugRelease Behavior of Poly (AA-co-PEGMEA)/Bentonite Nanocomposite Hydrogels for Mucoadhesive / W. Lee, Y. Chen // Journal of Applied Polymer Science. - 2004. -Vol. 91.P. 2934-2941.

[17] Adebola A. Oketola, Nelson Torto. Synthesis and characterization of poly (styrene-co-acrylamide) polymers prior to electrospinning. Advances in Nanoparticles, 2013, 2, 87-93.

[18] Ganiyev B. S., Sharipov M. S. Investigation of the Differential Thermodynamic Analysis of New Bifunctional Compositions Based on Navbahor Bentonites and Styrene-Acrylic Copolymers //Chemical and Biomolecular Engineering. 2020. - T. 5. - №. 1. - C. 35.

[19] Ganiyev B. Sh., Sharipov M. S. Study of the properties of natural sorbents and their modified forms. Materials of the Republican scientific-practical conference "Actual problems of Chemical Science and innovative technologies in its teaching". Tashkent 2016 y. - P. 159-161.

[20] Ruyi Chen, Fubing Peng, Shengpei Su. Synthesis and characterization of novel swelling tunable oligomeric poly(styrene-co-acrylamide) modified clays. Journal of Applied Polymer Science, 2008. Vol. 108, 2712-2717

[21] Anastasiadou, M., et al. "Polarimetric imaging for the diagnosis of cervical cancer." physica status solidi c 5.5 (2008): 1423-1426.

[22] Morton D. E., Johs B., Hale J. Optical monitoring of thin films using spectroscopic ellipsometry //PROCEEDINGS OF THE ANNUAL TECHNICAL CONFERENCE-SOCIETY OF VACUUM COATERS. - 2002. - C. 299-305.

[23] Hinrichs, K.; Furchner, A.; Rappich, J.; Oates, T. W. H., "Polarization-dependent and ellipsometric infrared microscopy for analysis of anisotropic thin films," The Journal of Physical Chemistry C 2013, 117 (26), 13557-13563, DOI: 10.1021/jp401576r.

[24] Furchner, A.; Bittrich, E.; Uhlmann, P.; Eichhorn, K.-J.; Hinrichs, K., "In-situ characterization of the temperaturesensitive swelling behavior of poly ( $\mathrm{N}$-isopropylacrylamide) brushes by infrared and visible ellipsometry," Thin Solid Films 2013, 541, 41-45, DOI: 10.1016/j.tsf.2012.10.135.

[25] Ganiyev B. Sh., Olimov B. B. Influence of synthesis temperature on the adsorption properties of polymer composites containing Navbahor bentonite / / Chemistry and chemical technology: achievements and prospects. - 2018. - P. 304.1-304.2.

[26] Ganiyev B. Sh., Sharipov M. S. Influence of initiator concentration on absorption properties polymer composite. IV All-Russian conference Chemistry and chemical technology: achievements and prospects. On November 27-28. Kemerovo. 2018. - P. 316.1.

[27] Ganiyev B. Sh., Sharipov M. S., Salimov F. G., Ikromov U. G. Influence of concentration of filler on process gel formation in the composition on the basis of bentonites and acrylic copolymers. International Journal of Advanced Research in Science, Engineering and Technology Vol. 6, Issue 10, October 2019. P. 11436-11440. 